\title{
INVENTARIO DE LAS PLANTACIONES DE EUCALIPTO DE LA VII REGION
}

\author{
R. Bennewitz, N. Vergara, J. Flores * \\ RESUMEN
}

Este trabajo entrega los resultados del inventario que el Instituto Forestal realizó en 1986 con el objetivo de prospectar las plantaciones de Eucalipto existentes en la VII Región, determinando la superficie y la ubicación de los rodales, el tipo de monte, la altura media y los volúmenes por hectárea.

Se aplicó la metodología tradicional que consiste en interpretar fotográficamente los límites de los rodales, obtener luego los antecedentes de terreno y, finalmente, elaborar la cartografía correspondiente y procesar los antecedentes numéricos.

Como resultados generales se logró determinar que el área de estudio contiene 4.301 há de plantaciones de Eucalipto distribuidas en 625 rodales; el $66 \%$ de la superficie de Eucalipto tiene entre 1 y 10 años de edad; el $69 \%$ de las plantaciones corresponden al tipo monte bajo, el $25 \%$ a monte alto y el $6 \%$ restante a monte medio; el volument total de madera en pie es de $851.564 \mathrm{~m}^{3}$, de los cuales el $52 \%$ está representado por el Eucalipto que crece en monte alto; el volumen medio de las plantaciones de Eucalipto de monte alto es de $466 \mathrm{~m}^{3}$ ssc por hectárea y el de las plantaciones de Eucalipto de monte bajo de $214 \mathrm{~m}^{3} \mathrm{ssc}$ por hectárea.

\section{ABSTRACT}

INFOR carried out an inventory in 1986 of the Eucalyptus plantations in Region VII, the findings of which are presented in this paper. The suney determined the area and location of the stands, type of forest, mean heights and volumes per hectare.

A traditional methodology was applied, which consists of interpreting photographically the boundaries of the individual stands, gathering data in the field and, finally, drawing the corresponding charts and maps, and processing the numerical data.

The study area was found to contain 4,031 ha of Eucalyptus plantations distributed amon 625 stands. Two thirds of the total area covered by Eucalyptus have between 1 and 10 years of age; $690 \%$ of the plantations correspond to coppice-grown stands (shoots grown from the stump). $25 \%$ to stands grown from seeds and $6 \%$ to mixed stocking. The total volume of timber amounts to $851,564 \mathrm{~m}^{3}, 54 \%$ of which is accounted for by stands grown from seeds. Mean volume for this type of Eucalyptus plantations is 466 solid cubic meters without bark per hectare, while for coppice-grown forests the mean volume amounts to 214 solid cu.m. withourt bark per hectare.

*Ing. Forestales, U. de Chile. División Inventarios Forestales, Instituto Forestal. Huérfanos 554, Piso 6. Santiago - Chile. 


\section{INTRODUCCION}

La creciente importancia que ha adquirido en el país la madera de Eucalipto, utilizada principalmente para fines industriales, ha despertado un gran interés por disponer de información actualizada acerca de la localización y características dasométricas de las masas existentes a nivel regional. El primer estudio destinado a satisfacer esta necesidad, realizado por el Instituto Forestal, a petición de la Corporación de Fomento de la Producción, fue el "Inventario de las Plantaciones de Eucalipto de la VII Región", cuyos resultados se dan a conocer en este trabajo.

En el marco de la planificación del uso de los recursos forestales, el estudio entrega antecedentes de gran interés para el desarrollo de políticas sectoriales, tanto a nivel estatal como privado. Complementariamente se exponen antecedentes técnicos necesarios para iniciar prácticas de manejo intensivo de las plantaciones de Eucalipto existentes en la VII Región, tales como tablas de volumen por árbol, rendimiento por hectárea, superficies y edades.

\section{OBJETIVOS}

El objetivo fundamental del inventario fue prospectar las plantaciones del género Eucalyptus existentes en la VII Región del país (Región del Maule), con el fin de obtener la ubicación geográfica de los rodales, superficies, tipo de monte, altura media y volumen por hectárea. Para estos efectos se establecieron además los siguientes objetivos secundarios:

a) Elaboración de cartografía forestal en escala 1:50.000 con la localización de todas las plantaciones del género Eucalyptus de tamaño igual o superior a 1 ha existentes en el área de estudio.

b) Cuantificación de la superficie cubierta por plantaciones del género Eucalyptus a nivel regional, provincial y comunal, en la VII Región.

c) Determinación del tipo de monte, altura media y diámetro medio de todos los rodales del género Eucalyptus de tamaño igual o superior a 5 ha existentes en la VII Región.

d) Construcción de tablas de volumen cúbico por árbol para plantaciones de Eucalyptus globulus de monte alto y monte bajo.

e) Estimación regional del volumen cúbico de madera en pie de las plantaciones del género Eucalyptus.

\section{ANTECEDENTES GENERALES DEL AREA DE ESTUDIO}

La VII Región del Maule se extiende aproximadamente entre las siguientes coordenadas geográficas: $34^{\circ} 45^{\prime}$ a $36^{\circ} 30^{\prime}$ de latitud Sur y $72^{\circ} 45^{\prime}$ a $70^{\circ} 30^{\prime}$ de longitud Oeste (Ver Figura 1).

La VII Región cubre una superficie de 3.051 .810 ha, que representan alrededor del $4 \%$ del territorio nacional (Instituto Geográfico Militar, 1981). Existen en la Región 1.968.800 ha de uso silvoagropecuario, de las cuales un $14 \%$ corresponde a suelos agrícolas, un $38 \%$ a suelos de aptitud ganadera y el $48 \%$ restante a suelos con aptitud de uso forestal (Universidad de Chile, Sede Talca, 1975).

En cuanto a su división administrativa, el área de estudio incluye cuatro provincias, que en conjunto poseen veintinueve comunas. 


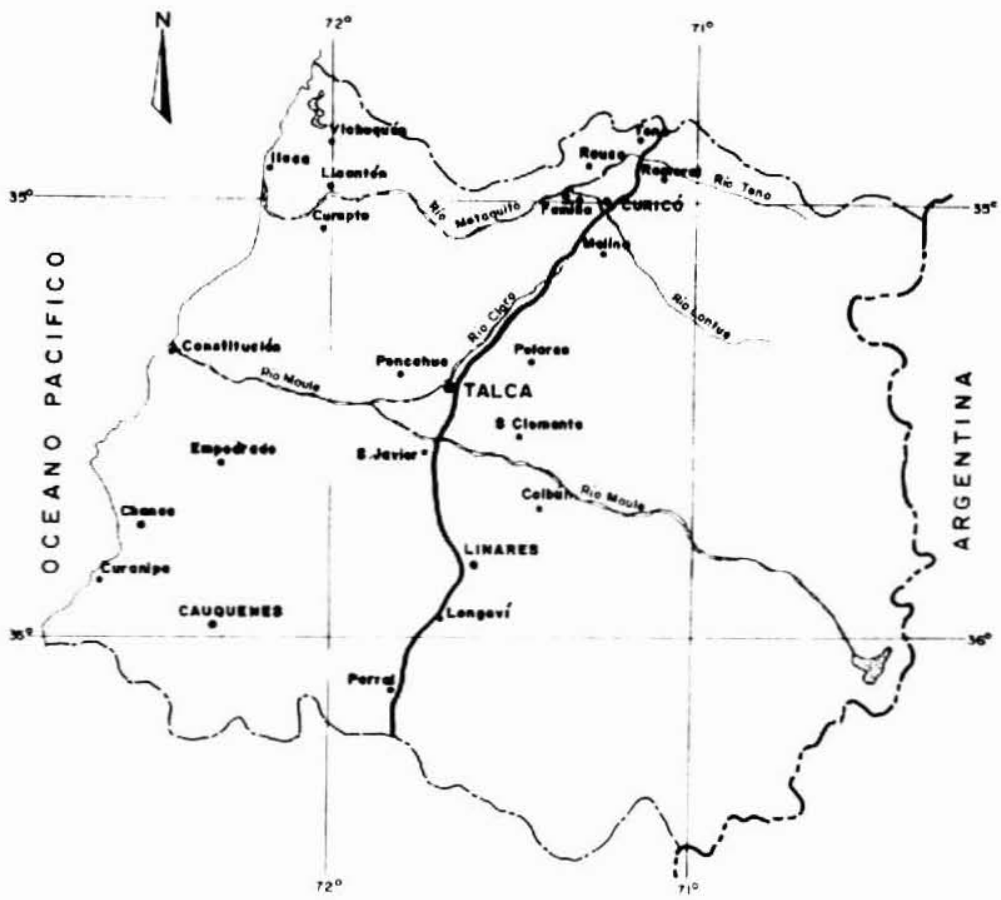

\section{MATERIAL Y METODO}

Material: Para la realización del presente estudio se utilizaron los siguientes materiales:

- Cartas topográficas elaboradas por el Instituto Geográfico Militar, en escala 50.000 .

- Mapas forestales de predios ubicados en la VII Región en los cuales existen plantaciones de Eucalipto. Estos mapas se recopilaron en las principales empresas forestales que poseen plantaciones en el área de estudio y en las oficinas provinciales de CONAF. En total se consultaron 66 mapas.

- Cartografía de roles de propiedad (según el Servivio de Impuestos Internos) en escala 1:100.000, procesada por CONAF VII Región, en base a los mosaicos de IREN en escala 1:20.000 y 1:50.000.

- Cartografía forestal obtenida como resultado del "Inventario de las Plantaciones Forestales de la VII Región", efectuado en 1981 por el Instituto Forestal para CIREN - CORFO.

- Fotografías aéreas pancromáticas blanco y negro, escala 1:30.000, Vuelo SAF $1978 / 79$. 
Método: La metodología utilizada por el estudio consultó la ejecución de las siguientes etapas:

1. Recopilación de antecedentes.

2. Fotointerpretación de las plantaciones de Eucalipto.

3. Trabajos de terreno.

- Control de la fotointerpretación.

- Mediciones básicas en todos los rodales de Eucalipto de tamaño mayor o igual a 5 ha.

- Muestreo de las plantaciones de Eucalipto.

- Obtención de una muestra para la elaboración de tablas de volumen por árbol.

4. Elaboración de cartografía forestal.

5. Procesamiento computacional electrónico de la información (Ver Figuras 2 y 3 ).

6. Elaboración de las Tablas de Volumen.

7. Procesamiento de la información referente a parcelas de muestreo.

8. Cálculo estadístico de las existencias volumétricas.

9. Obtención de cuadros de resultados.

FIGURA 2

DIAGRAMA DE FLUJO SIMPLIFICADO

DEL SUBSISTEMA DE PROCESAMIENTO

DE LA INFORMACION PROVENIENTE DE LAS PLANT ACIONES FORESTALES

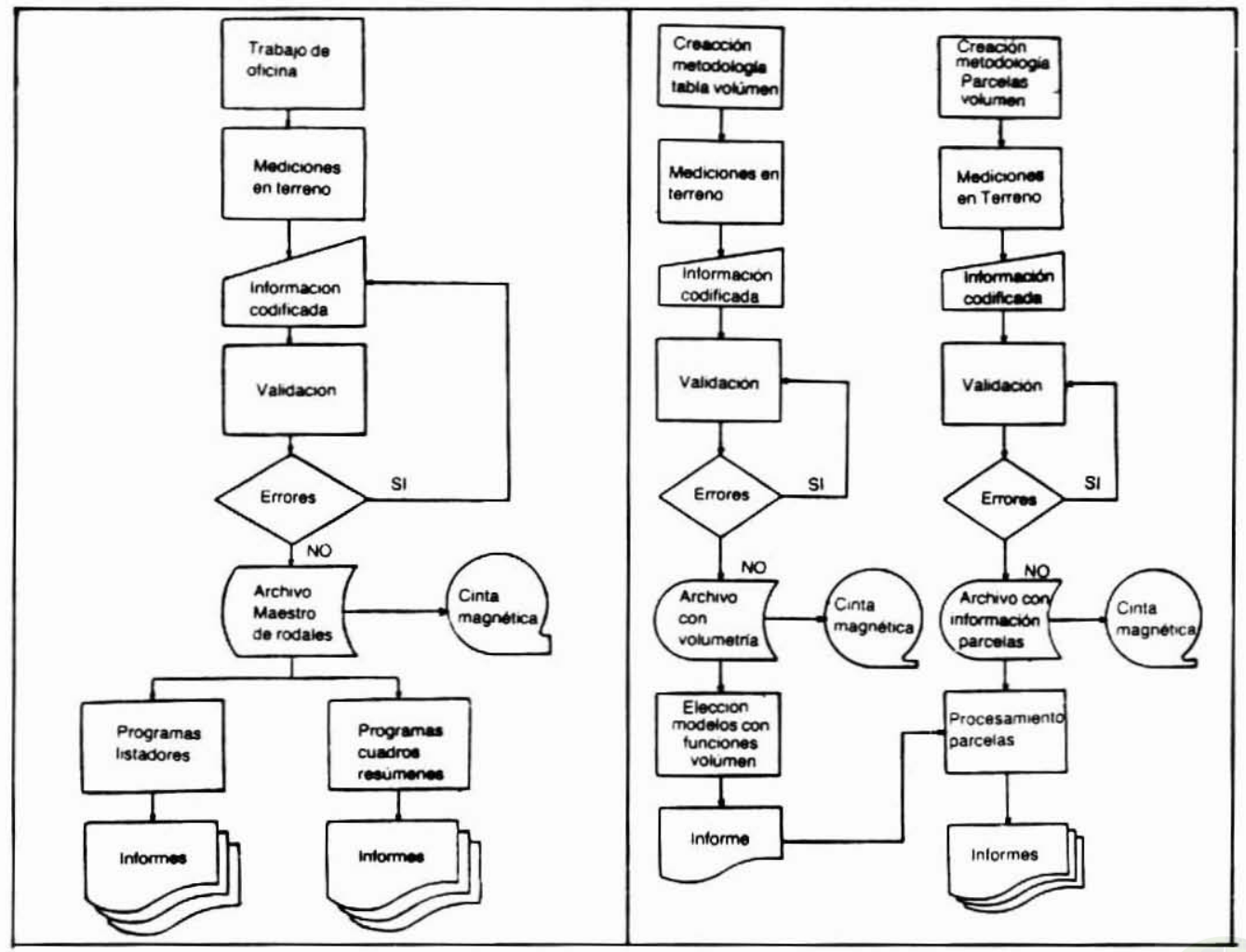

FIGURA 3

DIAGRAMA DE FLUJO SIMPLIFICADO DEL SUBSISTEMA DE TABLAS DE VOLUMEN Y UNIDADES MUESTRALES. 


\section{RESULTADO}

\section{Superficie}

- La superficie total de plantaciones de Eucalipto existente en la VII Región del Maule alcanza a $4.301,3$ ha, repartidas en un total de 625 rodales (Ver Cuadros 1 y 2).

- La provincia de Talca es la que concentra la mayor extensión de plantaciones de Eucalipto (2.244,6 ha), seguida por Curicó (1.289,3 ha), Linares $(456,1 \mathrm{ha})$ y Cauquenes $(311,3 \mathrm{ha})$.

- En la provincia de Talca, el 59,2\% de la superficie de plantaciones de Eucalipto se concentra en la comuna de Constitución. Las comunas de Río Claro y San Clemente poseen en conjunto el $36,8 \%$ de la superficie de plantaciones de Eucalipto existente en la provincia. No se detectó presencia de Eucalipto en las comunas de Curepto, Maule y Pencahue.

- En la provincia de Curicó, el 46,6\% de la superficie de plantaciones de Eucalipto se concentra en la comuna de Molina y el 28,4\% en la comuna de Curicó.

- En la provincia de Linares destacan por su importancia las comunas de Linares, Yerbas Buenas y Colbún. las cuales concentran respectivamente el $29,0 \%$, el $17,3 \%$ y el $15,0 \%$ de la superficie total de Eucalpto de la provincia.

\section{CUADRO 1 \\ SUPERFICIE DE PLANTACIONES DE EUCAUPTO POR EDAD Y TIPO DE MONTE VII REGION}

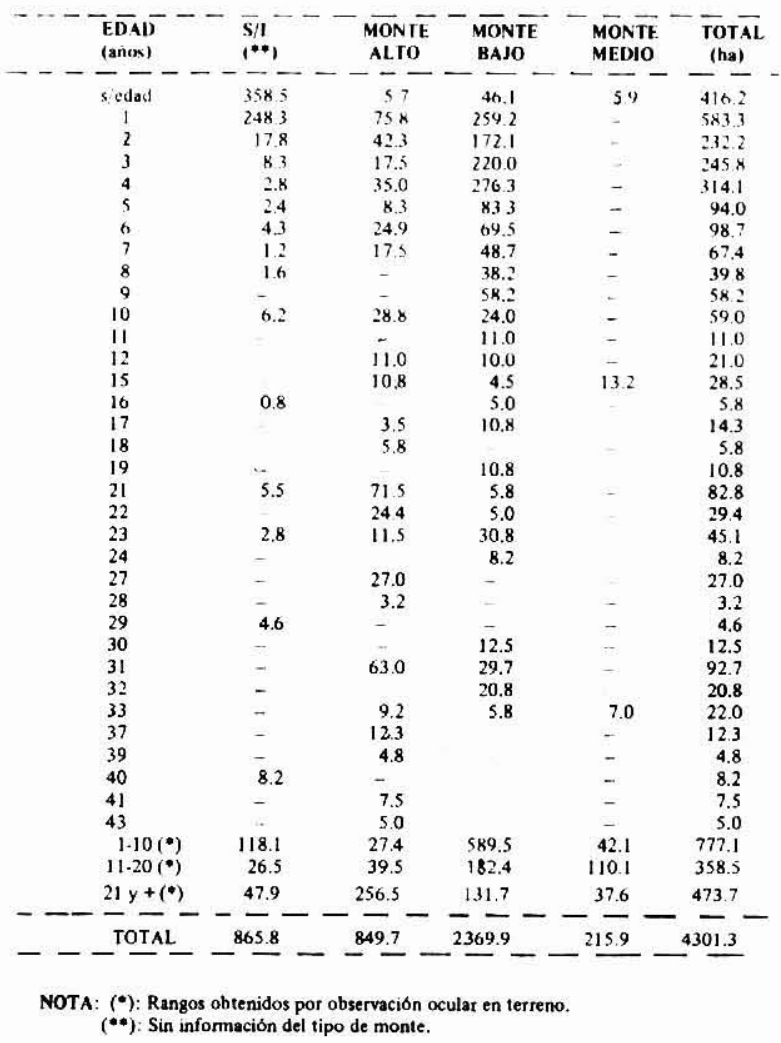


- En la provincia de Cauquenes, el 52,8\% de la superficie de plantaciones de Eucalipto se concentra en la comuna de Chanco y el $32,3 \%$ en Pelluhue.

- Las plantaciones de Eucalipto localizadas en el área agrícola de la Depresión Intermedia de la VII Región están sujetas en su mayor parte a prácticas de riego y rotaciones de corta duración.

- El $66,1 \%$ de la superficie con información de edad corresponde a plantaciones de Eucalipto con edades comprendidas entre 1 y 10 años, en tanto que el $11,7 \%$ y el $22,2 \%$ están representados respectivamente por recurso cuya edad oscila entre 11 y 20 años y entre 21 y más años de edad ( Gráfico 1).

\section{CUADRO 2}

\section{NUMERO DE RODALES DE EUCALIPTO SEGUN COMUNA Y TIPO DE MONTE VII REGION}

\begin{tabular}{|c|c|c|c|c|c|}
\hline \multirow[b]{2}{*}{ Comuna } & \multirow[b]{2}{*}{ S/I (*) } & \multicolumn{3}{|c|}{ Tipo de Monte } & \multirow[b]{2}{*}{ TOTAL } \\
\hline & & $\begin{array}{c}\text { Monte } \\
\text { Alto }\end{array}$ & $\begin{array}{c}\text { Monte } \\
\text { Bajo }\end{array}$ & $\begin{array}{l}\text { Monte } \\
\text { Medio }\end{array}$ & \\
\hline Curicó & 13 & 2 & 42 & - & 57 \\
\hline Hualañé & 2 & 2 & 3 & - & 7 \\
\hline Licantén & 1 & 1 & 4 & - & 6 \\
\hline Molina & 20 & 2 & 68 & - & 90 \\
\hline Rauco & - & - & 1 & - & 1 \\
\hline Romeral & - & - & 7 & 1 & 8 \\
\hline Teno & 2 & - & 2 & - & 4 \\
\hline \multicolumn{2}{|c|}{ Sagrada Familia 9} & 1 & 8 & - & 18 \\
\hline Vichuquén & - & 5 & 2 & - & 7 \\
\hline Constitución & 24 & 74 & 68 & 15 & 181 \\
\hline Empedrado & 1 & 1 & - & - & 2 \\
\hline Pelarco & 16 & - & 3 & - & 19 \\
\hline Río Claro & 9 & 5 & 16 & 1 & 31 \\
\hline Sn.Clemente & 25 & 2 & 27 & - & 54 \\
\hline Talca & 9 & - & 1 & - & 10 \\
\hline Colbún & 4 & 2 & 4 & - & 10 \\
\hline Linares & 9 & - & 13 & - & 22 \\
\hline Longaví & 3 & - & 4 & - & 7 \\
\hline Parral & 2 & 1 & 1 & - & 4 \\
\hline Retiro & 9 & 1 & - & - & 10 \\
\hline San Javier & 7 & - & - & 1 & 8 \\
\hline Villa Alegre & 6 & - & 5 & - & 11 \\
\hline Yerbas Buena & as 4 & - & 6 & - & 10 \\
\hline Cauquenes & 10 & 2 & 4 & - & 16 \\
\hline Chanco & 12 & 7 & 4 & 1 & 24 \\
\hline Pelluhue & 3 & 4 & - & 1 & 8 \\
\hline TOTAL & 200 & 112 & 293 & 20 & 625 \\
\hline
\end{tabular}

NOTA: $\left({ }^{*}\right)$ Número de rodales sin información del tipo de monte. 
- Las plantaciones de Eucalipto de la VII Región son fundamentalmente de monte bajo. El $69 \%$ de la superficie de plantaciones de Eucalipto con información de tipo de monte corres. ponde a monte bajo, en tanto que el $24,7 \%$ y el $6,3 \%$ están representados por plantaciones de monte alto y monte medio respectivamente (Ver Gráfico 2).

Régimen de Propiedad

- El 90\% de la superficie regional de las plantaciones de Eucalipto con información de propiedad está en manos del sector privado, en tanto que la propiedad estatal alcanza sólo al $10,0 \%$ de la superficie.

- El 94,3\% del número de rodales de Eucalipto con información de propiedad, pertenecen al sector privado, en tanto que sólo el $5,7 \%$ son de propiedad estatal.

\section{GRAFICO 1}

DISTRIBUCION PORCENTUAL POR CLASES DE EDAD DE LA SUPERFICIE Y NUMERO DE RODALES DE EUCALIPTO DE LA VII REGION

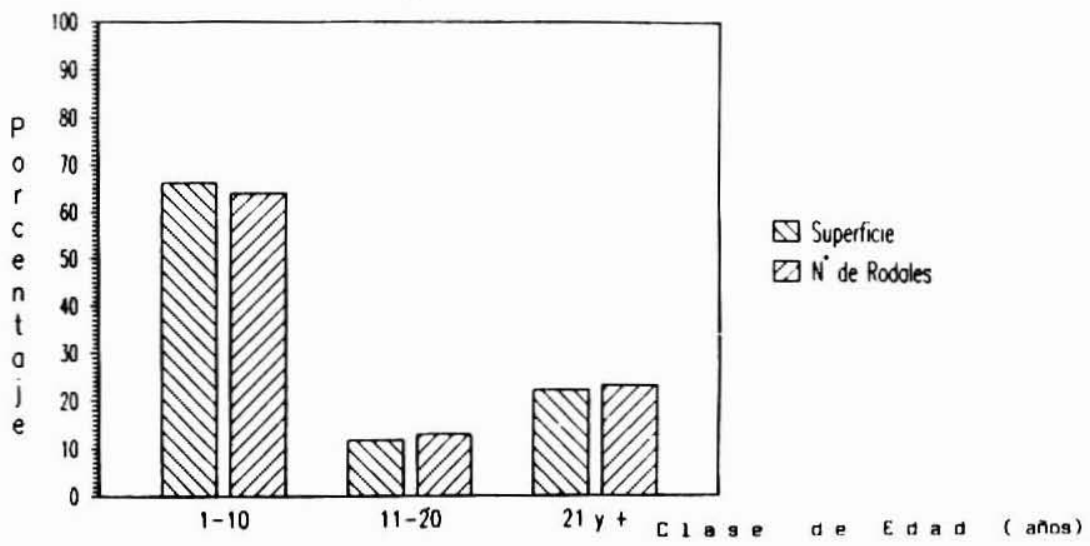

GRAFICO 2

DISTRIBUCION PORCENTUAL POR TIPO DE MONTE DE LA SUPERFICIE Y NUMERO DE RODALES DE EUCALIPTO DE LA VII REGION

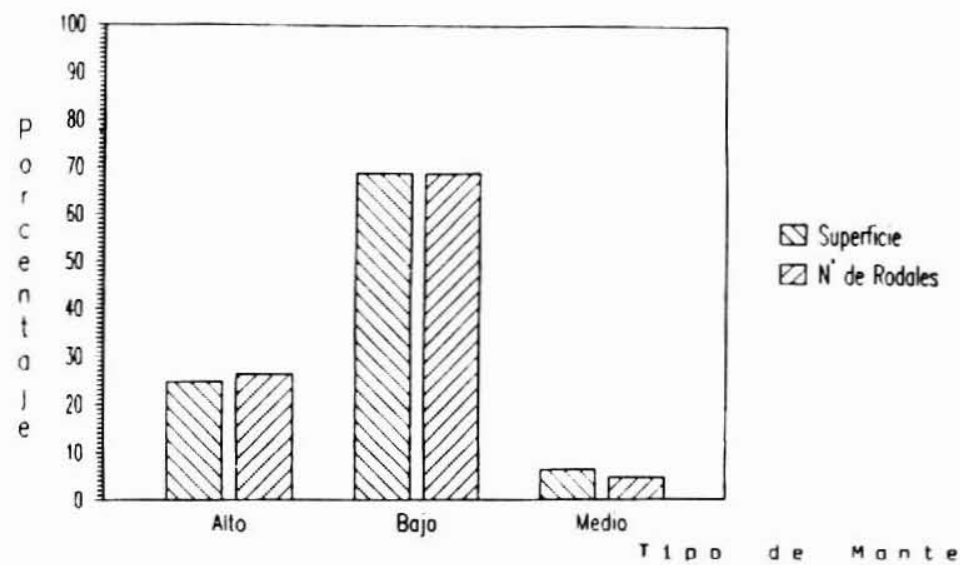


GRAFICO 3

DISTRIBUCION DEL NUMERO DE PROPIETARIOS Y DE LA SUPERFICIE TOTAL DE PLANT ACIONES DE EUCALIPTO CON INFORMACION DE PROPIEDAD, SEGUN

\section{CLASE DE TAMAÑO DE LA PLANTACION}

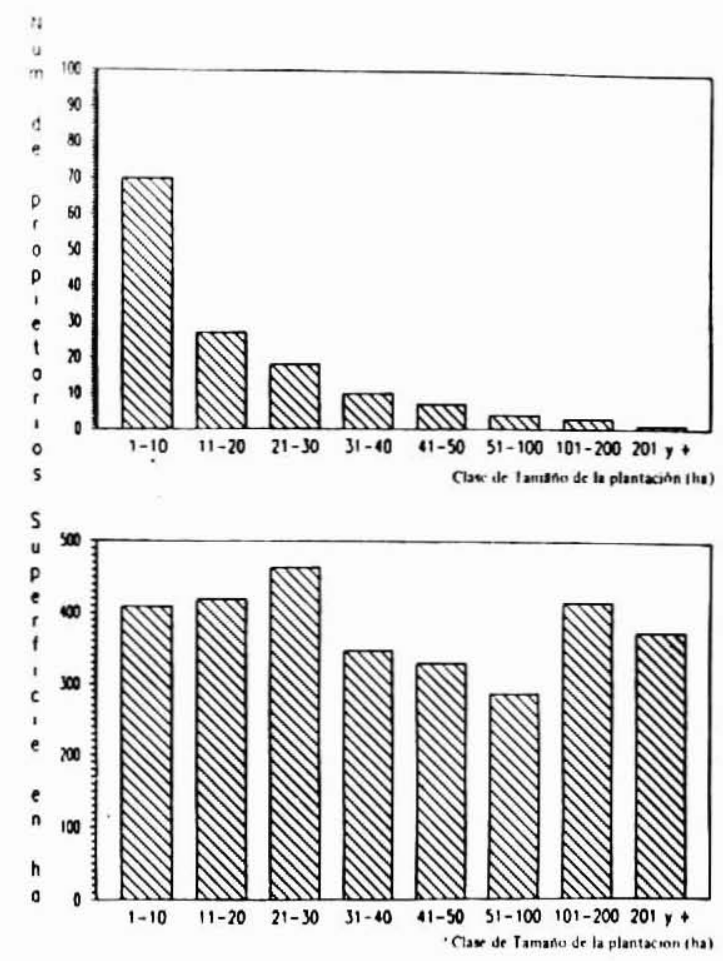

- El 70,7\% de la superficie total de plantaciones de Eucalipto (3.030,4 ha) está en manos de 140 propietarios, de los cuales la mitad poseen superficies de 1 a 10 ha de bosque. Aun cuando la cifra de pequeños propietarios es elevada, la superficie total que ellos poseen es relativamente pequeña, ya que sólo corresponde al $13,4 \%$ de la superficie con información de propiedad (Ver Gráfico 3).

- Las plantaciones de Eucalipto tienden a estar concentradas en pocos propietarios, ya que $1.402,6 \%$ ade la superficie regional están en manos de 15 propietarios, de los cuales 4 poseen en conjunto 787,7 ha ( Gráfico 3).

\section{Volumetría}

Tablas y Funciones de Volumen Cúbico por Arbol: El procedimiento de regresión paso a paso, junto con un análisis de los residuos de cada modelo, permitió desarrollar el siguiente modelo matemático:

$$
\ln \operatorname{VOL}=a+b \ln \left(\ln \left(\mathrm{DAP}^{2} \mathrm{H} T\right)\right)
$$

Los coeficientes del modelo y las características de cada ajuste se presentan en el Cuadro 3 . 


\section{CUADRO 3}

\section{FUNCIONES DE VOLUMEN CON Y SIN CORTEZA HASTA UN DIAMETRO} DE UTILIZACION DE $10 \mathrm{CM}$

\begin{tabular}{|c|c|c|c|c|c|c|c|}
\hline \multirow{2}{*}{$\begin{array}{l}\text { Tipo } \\
\text { Monte }\end{array}$} & \multirow{2}{*}{$\begin{array}{l}\text { Tipo } \\
\text { Volumen }\end{array}$} & \multicolumn{2}{|c|}{$\begin{array}{l}\text { Coeficientes de } \\
\text { Regresión }\end{array}$} & \multicolumn{4}{|c|}{$\begin{array}{l}\text { Indicadores de Grado } \\
\text { de Ajuste }\end{array}$} \\
\hline & & $\mathbf{a}$ & b & $\mathbf{r}$ & Syx & (\%) tb & $\mathbf{n}$ \\
\hline Alto & Con Corteza & $-22,9473$ & 9,8328 & 0,9884 & 17,2 & 77,4 & 143 \\
\hline Alto & Sin Corteza & $-23,5880$ & 10,019 & 0,9875 & 18,7 & 74,5 & 143 \\
\hline Вајо & Con Corteza & $-20,8000$ & 8,8864 & 0,9822 & 24,3 & 65,0 & 157 \\
\hline Bajo & Sin Corteza & $-20,1935$ & 8,5291 & 0,9865 & 23,0 & 75,3 & 157 \\
\hline
\end{tabular}

Donde:

VOL $=$ Volumen con o sin corteza hasta un diámetro de utilización de $10 \mathrm{~cm}\left(\mathrm{~m}^{3} /\right.$ árbol)

DAP = Diámetro a la altura del pecho $(\mathrm{cm})$

HT = Altura total del árbol $(\mathrm{m})$

$\mathrm{a}, \mathrm{b}=$ Coeficientes de regresión

$\mathrm{r} \quad=$ Coeficiente de correlación

Syx $(\%)=$ Error cuadrático medio $(\%)$

$t_{b} \quad=$ Valor " $t$ " de Student del coeficiente " $b$ "

$\mathrm{n} \quad=$ Número de observaciones

\section{GRAFICO 4 \\ FUNCIONES DE VOLUMEN SIN CORTEZA, POR TIPO DE MONTE}

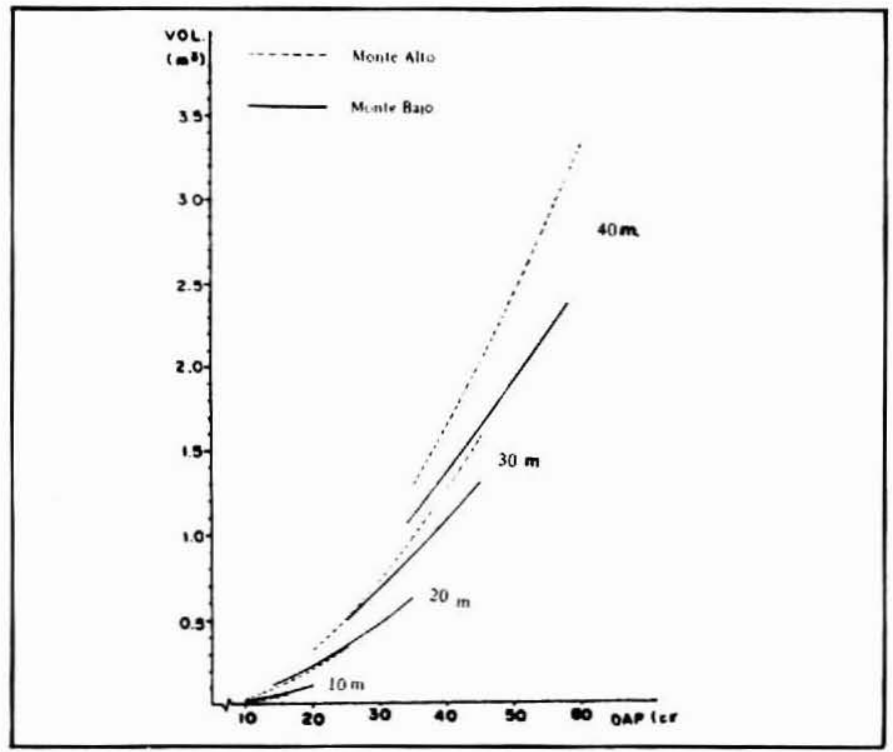

Volumen 1, Número 1, 1987/ 85 
Funciones de Volumen por Hectárea. La cubicación de las parcelas de muestreo de volumen permitió establecer relaciones entre el volumen, la altura y el área basal. En base a las tendencias obtenidas se elaboraron las siguientes funciones de volumen por hectárea:

$$
\begin{aligned}
& \text { VOL }=1.043,895\left(1-\mathrm{e}^{-0,075 \times \mathrm{xH}^{7,5840}}\right)^{7} \\
& \text { con } \mathrm{n}=87 \\
& r=0,8257 \\
& \operatorname{Syx}(\%)=40,5 \% \\
& \mathrm{VOL}=1.869,822\left(1-\mathrm{e}^{-0,012 \times \mathrm{AB}}\right)^{1,5519} \\
& \text { con } \mathrm{n}=87 \\
& \mathrm{r}=0,9682 \\
& \operatorname{Syx}(\%)=18,0 \% \\
& \operatorname{lnVOL}=-0,72950+0,9586 \ln (\mathrm{AB} \times \mathrm{H}) \\
& \operatorname{con} \mathrm{n}=87 \\
& r=0,9951 \\
& \operatorname{Syx}(\%)=16,7 \%
\end{aligned}
$$

Donde:

VOL : Volumen cúbico $\left(\mathrm{m}^{3} \mathrm{ssc} / \mathrm{ha}\right)$

$\mathrm{AB}$ : Area Basal ( $\left.\mathrm{m}^{2} / \mathrm{ha}\right)$

$\mathrm{H} \quad$ : Altura media del rodal $(\mathrm{m})$

Otras Relaciones Funcionales. La muestra de árboles utilizada para la elaboración de la tabla de volumen permitió obtener las siguientes relaciones funcionales para monte alto y monte bajo:

$$
\begin{aligned}
& \text { Monte alto }(\mathrm{n}=143 \text { observaciones }) \\
& \qquad \begin{array}{r}
\mathrm{HT}=48,9427\left(1-\mathrm{e}^{-0,055 \times \mathrm{xAP}}\right) \\
\mathrm{S}_{\mathrm{yx}}=0,7685 \\
\mathrm{HC}=-7,96,8 \%
\end{array} \\
& \qquad \begin{array}{r}
\mathrm{r}=0,9826 \\
\mathrm{~S}_{\mathrm{yx}}=6,3 \%
\end{array} \\
& \hline
\end{aligned}
$$




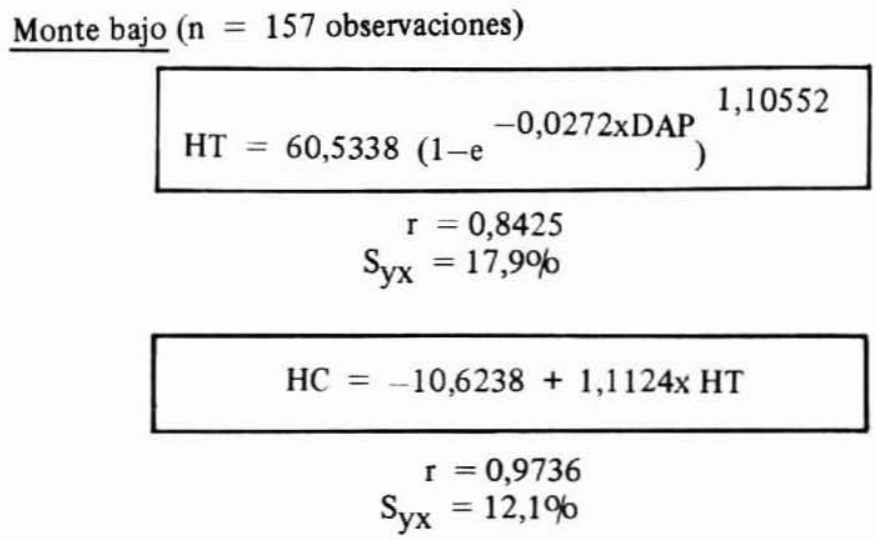

Donde:

HT : Altura total (m)

HC : Altura comercial para un diámetro de utilización de $10 \mathrm{~cm}(\mathrm{~m})$

DAP : Diámetro a la altura del pecho $(\mathrm{cm})$

\section{Existencias Volumétricas}

- El volumen total de madera en pie de las plantaciones de Eucalipto localizadas en la VII Región, hasta un índice de utilización de $10 \mathrm{~cm}$, alcanza a $851.564 \mathrm{~m}^{3}$ sólidos sin corteza.

- El error de muestreo del Volumen Medio Regional de madera en pie es de un 10,0\% para un coeficiente de confianza de $95 \%$. Considerando dicho error de muestreo, puede afirmarse que el volumen medio de madera en pie de las plantaciones de Eucalipto del área de estudio fluctúa entre 770 y 940 mil metros cúbicos sólidos sin corteza.

- De la existencia volumétrica regional, un 52\% corresponde a plantaciones de monte alto; la fracción restante equivalente al $48 \%$ del volumen, corresponde a monte bajo.

- El volumen medio por hectárea alcanza a $214 \mathrm{~m}^{3} \mathrm{ssc}$ en las plantaciones de monte bajo y a $466 \mathrm{~m}^{3} \mathrm{ssc}$ en las plantaciones de monte alto. 


\section{REFERENCIA BIBLIOGRAFICA}

CORPORACION NACIONAL FORESTAL 1985. Boletín Técnico No 25. Funciones de Volumen Cúbico para la Especie Eucalyptus globulus Labill, de Monte Bajo en la Región Metropolitana. Santiago, Chile. $74 \mathrm{p}$.

CORPORACION NACIONAL FORESTAL 1986. Boletín Técnico No 28. Tablas Generales de Producción de Leña para Eucalyptus globulus Labill en la Región Metropolitana. Santiago, Chile. 87 p.

FLORES, J. 1983. Proposición de una Metodología para Cubicar Arboles de Pinus radiata D. Don. Tesis Ing. For. Santiago, Universidad de Chile, Facultad de Ciencias Agrarias, Veterinarias y Forestales. Santiago. $62 \mathrm{p}$.

HERNANDEZ, M. y MORALES, H. 1985. Evaluación de la productividad de Eucalyptus spp. bajo diferentes condiciones de sitio VI a IX Región. Tesis Ing. For. Santiago, Universidad de Chile, Facultad de Ciencias Agrarias, Veterinarias y Forestales. Santiago. 131 p.

INSTITUTO DE RECURSOS NATURALES 1979. Perspectivas de Desarrollo de los Recursos e la VII Región. Publicación No 25. Santiago, Chile, IREN - CORFO. Tomo X: GEODEMOGRAFIA y Tomo VIII (Análisis de los Recursos Forestales).

INSTITUTO FORESTAL 1986. Estadísticas Forestales 1985. Santiago, Chile, INFOR-CORFO, Serie Informática No 34 , junio $1986,98 \mathrm{p}$.

INSTITUTO GEOGRAFICO MILITAR 1981. Atlas Regionalizado de Chile. Santiago, Chile, Instituto Geográfico Militar. 2da. Edición.

INSTITUTO GEOGRAFICO MILITAR 1983. Geografía de Chile, V. 3: Biografía. Santiago, Chile. Instituto Geográfico Militar. 230 p.

INSTITUTO GEOGRAFICO MILITAR 1983. Atlas de la República de Chile. Santiago, Chile, Instituto Geográfico Militar, 2da. Edición. 349 p.

LEHMAN, W. WALTER 1967. Construcción de Tablas de Volumen para Eucaliptus globulus Labill en la zona costera Centro-Norte. Tesis Ing. For. Valdivia, Universidad de Chile, Escuela de Ingeniería Forestal. $69 \mathrm{p}$.

LISBOA, H. 1960. Construcción de Tablas de Volumen para Eucalyptus globulus Labill. Tesis Ing. For. Santiago, Universidad de Chile, Facultad de Ciencias Forestales, 45 p.

LUTTECKE, KLAUS 1983. Análisis Técnico y Económico de Mediciones Lineales del Fuste por Medio del Relascopio de Banda Ancha de Bitterlich. Universidad de Chile. Facultad de Ciencias Agrarias, Veterinarias y Forestales, Santiago. 58 p.

RIBALTA, E. 1983. Evaluación de la Producción y Productividad del Monte Bajo de Eucalyptus globulus Labill V Región. Tesis Ing. Forestal, Santiago, Universidad de Chile. Facultad de Ciencias Agrarias, Veterinarias y Forestales, $128 \mathrm{p}$.

SEPULVEDA, S. 1962. Síntesis Regional en: Geografía Económica de Chile. Tomo IV. Corporación de Fomento. Editorial Universitaria. Santiago, Chile.

UNIVERSIDAD DE CHILE - SEDE TALCA 1975. Primera y Segunda Jornadas de Recursos Naturales Renovables de la Región del Maule. Acta Reunión Final. 